\title{
Comparison of Blood Clot Culture with Conventional Blood Culture and Biphasic Blood Culture for the Diagnosis of Enteric Fever
}

\author{
T. Sabeetha ${ }^{1}$, A.V.M. Balaji ${ }^{2}$ and J. Nithyalakshmi ${ }^{3 *}$ \\ ${ }^{1}$ Institute of Venerology, Madras Medical College, Chennai, Tamil Nadu, India \\ ${ }^{2}$ Stanley Medical College, Chennai, Tamil Nadu, India \\ ${ }^{3}$ Sri Muthukumaran Medical College and Research Institute, Mangadu, Tamil Nadu, India \\ *Corresponding author
}

\begin{abstract}
A B S T R A C T
Keywords

Diagnosis of enteric fever based on clinical presentation is difficult as signs and symptoms are often non-specific. Isolation of enteric fever pathogens remains the gold standard in diagnosis. Removal of serum with antibacterial activity in clot culture, may enhance the

Blood clot culture,

Blood culture, growth of typhoid bacilli. We would like to evaluate clot blood culture system over conventional blood culture and Biphasic blood culture for diagnostic accuracy. Blood cultures by conventional blood culture, Biphasic blood culture and clot culture were

Diagnosis performed for 100 consecutive patients for whom enteric fever is suspected. The

Article Info

Accepted:

14 December 2017

Available Online: sensitivity and specificity of whole blood culture and Biphasic medium was found to be $72 \%$ and $100 \%$.Both system were of equal sensitivity. Clot culture showed $100 \%$ sensitivity. From our study, we strongly recommend clot bile culture be used for routine diagnostic purpose in areas where enteric fever is endemic. Major advantage of practicing clot culture is we utilize what is usually considered as leftover, avoiding the need for additional volume of blood and found to have increased potential for isolation.
\end{abstract}

\section{Introduction}

Enteric fever remains as an important cause of morbidity despite advances in diagnostic technology and public health strategy. According to the most recent estimates by WHO, approximately 2,00,000 people die every year worldwide.

The bulk of the burden being borne by Asian countries as it accounts for $90 \%$ of this mortality (Amicizia, 2017). Enteric fever is caused by Salmonella enterica serovar Typhi (S. typhi), S. paratyphi A and, less commonly, by $S$. paratyphi B (Schotmulleri) and $S$. paratyphi $\mathrm{C}$ (Hirschfeldii).

Diagnosis of enteric fever based on clinical presentation is difficult as signs and symptoms are often non-specific. The most reliable means of confirming an infection in an endemic area is isolation of serotype Typhi from blood, urine, or stool.

There are technical and practical limitations for bone marrow culture which is still considered to be the gold standard for definite diagnosis (Christopher, 2011). 
Delay in diagnosis and inappropriate treatment may result in the emergence of drug resistance Salmonella Typhi. Resistance to common first line antibiotics and quinolones by Salmonella Spp., has complicated the management of typhoid fever and heightened the need for accurate at the same time, prompt diagnosis of enteric fever.

Accurate diagnosis can be made by isolating the organism by the Blood culture, the most standard diagnostic method. (Lee, 2004)

In our hospital settings, isolation can be done by conventional blood culture method, clot culture and Biphasic blood culture.

In clot culture, serum with bactericidal activity has been removed well before culturing clot. This would probably enhance the growth of typhoid bacilli.

It was reported to be more sensitive than other blood culture system. We considered clot culture as gold standard and compared it with other systems. Biphasic culture medium has an advantage that subculture could be done by tilting, reducing the risk of contamination.

We would like to evaluate clot blood culture system over conventional blood culture and Biphasic blood culture for diagnostic accuracy.

\section{Materials and Methods}

The present study was aimed to evaluate the performance of blood clot culture over whole blood culture in BHI broth and in BHI Broth in Trypticase Soy Agar

Biphasic Medium. The study was carried out in the department of Microbiology, in a teaching tertiary care Hospital, Chennai. Institutional Ethical Committee clearance was obtained.

\section{Inclusion criteria}

100 consecutive patients clinically suspected for enteric fever presenting with duration of fever less than a week, were included in this study. Informed consent was obtained before collecting the blood specimen.

\section{Exclusion criteria}

Patients with known cause of fever, like UTI, Tuberculosis, Malaria, AIDS were excluded.

History of fever more than a week.

Under strict aseptic precaution, $20 \mathrm{ml}$ of venous blood was collected in the first week of clinically suspected cases of enteric fever before starting the antibiotics and processed in the following methods.

About 20ml of blood sample is collected under strict aseptic precautions in the first week of clinically suspected cases of enteric fever before starting the antibiotics and processed in the following methods.

\section{Conventional blood culture method with BHI broth}

About $10 \mathrm{ml}$ of blood is inoculated into $50 \mathrm{ml}$ of BHI broth (1: 5 dilution) and incubated at $37^{0}$ C. After 48 hours, subculture onto MacConkey agar, blood agar and Nutrient agar and incubated at $37^{\circ} \mathrm{C}$. If no growth occurs, subculture everyday till seven days.

\section{Clot culture method}

About $5 \mathrm{ml}$ of blood is collected in a sterile container and left at room temperature for two hours. After separating the serum aseptically, the clot was lysed with sterile glass rod and inoculated in $\mathrm{Ox}$ bile broth and incubated at $37^{0}$ C. After 48 hours subcultured onto MacConkey agar, blood agar, and Nutrient 
agar and incubated $37^{\circ} \mathrm{C}$. If no growth occurs, subcultured everyday till seven days.

\section{Biphasic blood culture method with BHI broth and Trypticase soy agar}

About $5 \mathrm{Ml}$ of blood is inoculated into $\mathrm{BHI}$ broth in Trypticase Soy Agar biphasic medium and incubated at $37^{\circ} \mathrm{C}$.

Tilt the biphasic medium container in such a way that the broth tilted over the slant and incubated further in erect position and observe for growth daily. If no growth occurs, observes everyday till seven days.

The date of appearance of growth in both culture systems was recorded for comparison of growth rates.

Following isolation of Salmonella spp., they were identified by standard microbiological methods. Antibiotic susceptibility test was performed in accordance with CLSI guidelines using Ampicillin, Ciprofloxacin, Chloramphenicol, Cotrimoxazole, Tetracycline and Ceftriaxone discs (CLSI, 2017).

Statistical analysis: Data were entered into an Excel spreadsheet. Accuracy of conventional blood culture and Biphasic medium was determined in terms of Sensitivity, specificity, positive predictive value (PPV), and negative predictive value (NPV) using $2 \times 2$ table. Results of these two systems were compared with Clot culture.

\section{Results and Discussion}

Blood culture by all three techniques were performed and 11 cultures were positive by one or the other method. Out of 11, 7 $(63.63 \%)$ and $4(36.36 \%)$ were found to be $S$. typhi and S.paratyphi respectively (Fig. 1). Clot culture was positive in all 11 cases, there were no single specimen where clot was negative but other system was positive for growth. Overall, culture positivity for Clot, Blood and Biphasic was 100\%, 72.72\% and $72.72 \%$ (Table 1). Isolation time for majority of the isolate $(90.90 \%)$ was found to be $48 \mathrm{hrs}$ by clot culture whereas only $54.54 \%$ of isolates were grown by other two systems at the same incubation time (Table 2). We considered clot culture as gold standard and compared it over whole blood and biphasic medium. Sensitivity and Specificity of whole blood culture and Biphasic medium was found to be $72.73 \%$ and $100 \%$ (Table 3-5).

Enteric fever, a systemic infection caused by Salmonella enterica serotype Typhi (S. Typhi) and $S$. enterica serotpe Paratyphi $(S$. paratyphi) $\mathrm{A}, \mathrm{B}$, and $\mathrm{C}$ is an important cause of avoidable mortality. Our data indicate that clot bile culture is excellent in primary isolation of Salmonellae.

In our study, isolation rate of $S$. Typhi and $S$. Paratyphi A was $63.6 \%$ and $36.36 \%$ respectively. This is in accordance with the findings of Krishnan et al., (2009), who had reported $70 \%$ and $30 \%$ of isolates were $S$. typhi and $S$. paratyphi. In a similar study at Chennai, $76 \%$ and $24 \%$ were observed to be $S$. typhi and $S$. paratyphi which was also in accordance with our findings (Renu Mathew et al., 2013).

Among the 100 patients studied, clot bile culture confirmed 11 patients as enteric fever. Hence, culture positivity was $11 \%$.This observation is slightly higher than prevalence of $5.5 \%$ as reported by Peerepur et al., in his similar study.

In contrast, high prevalence of $34 \%$ was observed by Renu Mathew et al., (2013) Geographical and time based variations may probably responsible for this wide difference in prevalence. 


\section{Fig 1:Distribution of enteric fever pathogens}

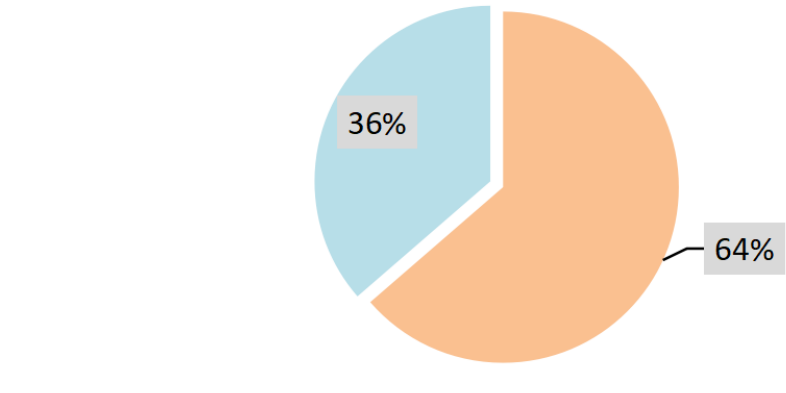

S.Typhi S.paratyphi

Table.1 Isolation rate of enteric fever Salmonella spp from each of three blood culture systems

\begin{tabular}{|c|c|c|c|}
\hline \multirow{2}{*}{ Organisms isolated } & \multicolumn{3}{|c|}{ No of specimens' positive (\%) } \\
\cline { 2 - 4 } & BHI & Biphasic - TSA & Clot culture \\
\hline S.Typhi & $5(45.45)$ & $6(54.54)$ & $7(63.63)$ \\
\hline S.paratyphi & $3(27.27)$ & $2(18.18)$ & $4(36.36)$ \\
\hline Total & $8(72.72)$ & $8(72.72)$ & $11(100)$ \\
\hline
\end{tabular}

Table.2 Recovery rate of enteric fever pathogens at various incubation period

\begin{tabular}{|l|l|l|l|l|}
\hline S.no & Incubation time & BHI & Biphasic with TSA & Clot culture \\
\hline $\mathbf{1}$ & Within 24 hrs & 0 & 0 & 0 \\
\hline $\mathbf{2}$ & $24-48 \mathrm{hrs}$ & 6 & 6 & 10 \\
\hline $\mathbf{3}$ & > 48 hrs & 2 & 2 & 1 \\
\hline
\end{tabular}

Table.3 Clot culture vs Conventional BHI blood culture

\begin{tabular}{|c|c|c|c|c|}
\hline & \multicolumn{2}{|c|}{ Clot culture } & \multirow[t]{2}{*}{ Total } \\
\hline & & Positive & Negative & \\
\hline \multirow{2}{*}{$\begin{array}{c}\text { Conventional BHI blood } \\
\text { culture }\end{array}$} & Positive & 8 & 0 & 8 \\
\hline & Negative & 3 & 89 & 92 \\
\hline \multicolumn{2}{|l|}{ Total } & 11 & 89 & 100 \\
\hline
\end{tabular}

Table.4 Clot culture vs Biphasic medium with TSA

\begin{tabular}{|c|c|c|c|c|}
\hline & \multicolumn{2}{|c|}{ Clot culture } & \multirow[t]{2}{*}{ Tota } \\
\hline & & Positive & Negative & \\
\hline \multirow{2}{*}{$\begin{array}{c}\text { Biphasic medium with } \\
\text { TSA }\end{array}$} & Positive & 8 & 0 & 8 \\
\hline & Negative & 3 & 89 & 92 \\
\hline \multicolumn{2}{|l|}{ Total } & 11 & 89 & 100 \\
\hline
\end{tabular}


Table.5 Parameters of interest to compare biphasic and conventional blood culture medium

\begin{tabular}{|l|l|l|}
\hline Parameters & Biphasic medium & Conventional blood culture \\
\hline Sensitivity & $72.73 \%$ & $72.73 \%$ \\
\hline Specificity & $100 \%$ & $100 \%$ \\
\hline Positive predictive value(PPV) & $100 \%$ & $100 \%$ \\
\hline Negative predictive value(NPV) & $96.74 \%$ & $96.74 \%$ \\
\hline
\end{tabular}

Whole blood culture and Biphasic culture was positive in 8 cases whereas clot bile culture was positive in 11 cases. Bile effectively inactivates anti-Salmonella activity of blood. Hence, several studies reported it to be superior to enriched non selective broths for isolation of enteric fever Salmonella spp. from whole blood (Escamilla et al., 1986 and Watson et al., 1954).

The data from our study are in agreement with this finding, showing that clot bile system is more sensitive in isolation compared to other systems. As 3 cases isolated only by clot bile remained negative by other systems even after 2 weeks of subculture.

$90.90 \%$ (10) of total isolates of clot bile culture grew within $48 \mathrm{hrs}$.In contrast, $54.54 \%$ (6) of whole blood and Biphasic medium isolates had grown at the same incubation period. Bacterial growth in clot bile culture was faster than other culture systems this was in accordance with other study (Mantur et al., 2007).

The sensitivity and specificity of whole blood culture and Biphasic medium was found to be $72.73 \%$ and $100 \%$. Both system were of equal sensitivity. Clot culture showed 100\% sensitivity, it was observed to be more sensitive than other systems.

In a similar study by Mantur et al., (2007) clot culture was found to be much more sensitive than whole blood. The results of the above study correlated well with our study.
From our study, we strongly recommend clot bile culture be used for routine diagnostic purpose in areas where enteric fever is endemic. Major advantage of practicing clot culture is we utilize what is usually considered as leftover, avoiding the need for additional volume of blood and found to have increased potential for isolation.

\section{References}

Amicizia D, Arata L, Zangrillo F, Panatto D, Gasparini R. Overview of the impact of Typhoid and Paratyphoid fever. Utility of Ty21a vaccine (Vivotif). Journal of Preventive Medicine and Hygiene. 2017;58(1):E1-E8.

Christopher M Parry, Lalith Wijedoru, Amit Arjyal \& Stephen Baker. The utility of diagnostic tests for enteric fever in endemic locations Expert Review of Anti-infective Therapy 2011; Vol 9(6), 711-725.

Escamilla J, Santiago LT, Sangalang RP Comparative study of three blood culture systems for isolation of enteric fever Salmonella. The Southeast Asian Journal of Tropical Medicine and Public Health [01 Jun 1984, 15(2):161-166].

Escamilla J, Ugrate HF, Kilpatrick ME. Evaluation of blood clot cultures for isolation of S. typhi, S. paratyphi A and Brucella melitensis. Journal of Clinical Microbiology 1986; 24(3):388-90.

Krishnan P, Stalin M, Balasubramanian S. Changing trends in antimicrobial resistance of Salmonella enterica serovar typhi and Salmonella enterica 
serovar paratyphi A in Chennai. Indian J Pathol Microbiol. 2009 Oct-Dec; 52(4):505-8.

Lee K, Yong D, Yum JH, et al., Emergence of Multidrug-Resistant Salmonella enterica Serovar Typhi in Korea. Antimicrobial Agents and Chemotherapy. 2004; 48(11):41304135.

Mantur BG, Bidari LH, Akki AS, Mulimani MS, Tikare NV, Diagnostic yield of blood clot culture in the accurate diagnosis of enteric fever and human brucellosis. Clin Lab. 2007; 53(1-2):57-
64.

NCCLS, 2017 National Committee for Clinical Laboratory Standards. Performance Standards for Antimicrobial Disk Susceptibility Tests. 27th Edn., Approved standard M2-A8. National Committee for Clinical Laboratory Standards, Wayne, Pa, USA.

Renu Mathew, Jobin. S.R.A comparative study on methods for diagnosis of enteric fever Int J Cur Res Rev, 2013; vol. 05 (14).

Watson, K. C. 1954. Clot culture in typhoid fever. J. Clin. Pathol. 7:305-307.

\section{How to cite this article:}

Sabeetha, T., A.V.M. Balaji and Nithyalakshmi, J. 2018. Comparison of Blood Clot Culture with Conventional Blood Culture and Biphasic Blood Culture for the Diagnosis of Enteric Fever. Int.J.Curr.Microbiol.App.Sci. 7(01): 1829-1834.

doi: https://doi.org/10.20546/ijcmas.2018.701.222 